\title{
Evaluating Gaze and Touch Interaction and Two Feedback Techniques on a Large Display in a Shopping Environment
}

\author{
Angelique Kessels, Evert van Loenen, and Tatiana Lashina
}

Philips Research, User Experiences,

High Tech Campus 34,

5656 AE Eindhoven

\{Angelique.Kessels, Evert.van.Loenen, Tatiana.Lashina\}@Philips.com

\begin{abstract}
At Philips Research, an Intelligent Shop Window (ISW) was developed. With the ISW, a unique combination is made between a transparent display that is embedded in the window glass, and physical products presented in the shop window. In this paper, touch and gaze interaction with the large display in the ISW are investigated and evaluated. For sensing interfaces it is known that especially with gaze interaction, it is a challenge to communicate to the user that the system is ready and attending to the user, but also which part of the system the user can address. Therefore, two suitable feedback mechanisms for this interaction were designed and evaluated with users. The first was the 'polite products' concept, where products were placed on a turntable. When the user selects the product with either touch or gaze interaction, the product on the turntable turns towards the user. The second feedback method was a transparent light tile behind the products, which changed color when the product was selected. The evaluation results showed that the polite product concept was rated better than the light tile on almost every item related to hedonic values such as enjoyability and fun. Next to that it became clear that participants felt more in control when using touch interaction and that touch worked faster and more efficient than gaze interaction. However, gaze interaction was a fairly new and exotic interaction method for participants and they said they liked using gaze interaction. Especially the combination of gaze interaction with the polite products feedback method was very strong.
\end{abstract}

Keywords: Touch interaction, gaze interaction, feedback mechanisms, large displays, public space.

\section{Introduction}

To investigate the use and benefits of new technologies in the public domain and specifically in shops, Philips has built the experience lab "Shoplab" on the High Tech Campus in Eindhoven. This Shoplab embodies a fashion shop where many prototypes 
that have associations with shopping are built in and can be tested with users. Shoplab also has a shop window [2, 3], which carries the name: "Intelligent Shop Window" (ISW). The Intelligent Shop Window responds to the presence, movement and input of shoppers near to the window, with light, sound, text, images and animations. It is also an interactive shop window where shoppers can obtain information about the products on display, using different interaction styles.

The research described in this paper focuses on the interactive part of the Intelligent Shop Window. Our first exploratory research question therefore is: What is the best way to interact with a large shop window? All interaction styles need to trigger some sort of feedback to clarify that the system responds to the input of the user. So from the first research question, a second one automatically arises: What is the best form of feedback that gives the user a clear and understandable interaction experience?

\section{Concept Design of the Intelligent Shop Window}

The sensors in the sensing environment around the ISW can be used as input devices for a user interface on the shop window. This way the interface can attend to what users need without getting explicit input from users [4]. These kind of interfaces are called attentive interfaces: user interfaces that dynamically prioritize the information they present to their users such that information processing resources of both user and system are optimally distributed across a set of tasks [5]. The design of the interface of the ISW is based on the attentive interface principle. Therefore we adopt the following hypothesis: in a shopping environment, an attentive interface on the shop window can attract customers, by dynamic adaptations to the information that is visible on the shop window. To this end, a differentiation will have to be made in various phases and states the shop window can be in. When a shopper is far away from the shop, the information on the shop window will obviously be different of content and size than when the user is near to the window. Also, it is essential that only information is given on products that are of interest to the user. When the user is very near to the shop window it is possible to see where he is looking at and only give information about that particular product. This implicit input should result in enhancing the appeal of the product and increasing the interest of the user.

\subsection{Interaction Design}

The interaction concept with the Intelligent Shop Window is based on the concept presented in [1]. Vogel et al. [1] present 4 interaction phases, related to distance of the user to the screen and body/head posture (figure 1):

- Ambient Display

- Subtle Interaction

- Implicit Interaction

- Personal Interaction 


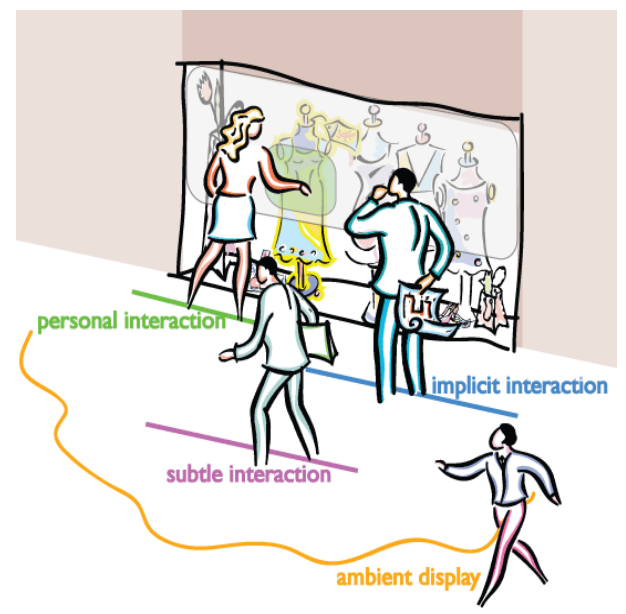

Fig. 1. Four phase interaction concept

The differentiation between the various phases is highly applicable to the ISW and will be further explained in the next paragraphs. Unfortunately Vogel et al. [1] only discuss the use of a screen with touch interaction and body orientation as implicit interaction with the system, while with the ISW the choice can be made between various sensors that can function as input devices, i.e. gaze, touch, position and direction of movement of the user. Also the products inside the shop window, combined with the screen provide an added dimension. Various adjustments to the implementation of the 4 phases are made and implemented in the ISW [2, 3].

\subsection{The Ambient Display}

The first interaction phase is the 'ambient display' phase according to Vogel et al. [1]. In the current ISW this is implemented as the 'attractor mode' of the window. This is the neutral stage of the screen when no shoppers are near to the shop. The screen is displaying the shop atmosphere and the message the shop wants to convey to the shopping people outside. Next to the current implementation of atmosphere creation, the system can show a range of information items like upcoming fashion shows, news updates and perhaps sale or discount information. A shopper that is walking by at a relative large distance should be able to get a sense of the overall information that is displayed at one glance.

\subsection{Subtle Interaction}

When a user walks parallel to the window, the system reacts to that. In the current implementation of the ISW, butterflies and text pop up on the screen at the position of the shopper. Also a range of audio devices produce a stream of directed audio. Both audio and visible attractors are moving along with the shopper as he walks along the shop window. The position and orientation of a user passing by or standing still in 
front of the shop window is determined by the use of a pressure sensitive floor in front of the ISW.

\subsection{Implicit Interaction}

When the user looks into the shop window, it is possible to track his gaze with cameras that are inside the shop window. In the current ISW, the gaze tracker gives the user the possibility to just look at an item in the shop and the system will give appropriate feedback on this product selection. To investigate which feedback is most appropriate, four feedback mechanisms are developed to give this feedback: the first feedback is information about the selected product, displayed on the screen of the shop window. Second, an audio feedback is given by a clearly audible 'click'. The third and fourth feedback mechanisms are developed to make the product more attractive: the 'polite products' concept and the 'light 'em up' concept.

\section{Feedback method: Polite products}

In the polite products concept, all products in the shop window are placed on a specially designed turntable. This turntable can rotate and tilt the product in any desired direction. The metaphor behind the concept is based on human behavior while having a conversation. When people talk to each other, they turn their body or at least their head towards the person they are talking to. It is often considered as polite to turn towards your speaking partner, so in the same manner, the products in the shop window will turn their 'faces' towards the customer when he/she looks at them. When the product is placed below the viewers eyes, the product will also be tilted, to give the customer the best view.

For the polite products concept, there were three design constraints:

1. The product in the shop window should be able show itself and turn towards the shopper who is in front of the window

2. The product can be placed anywhere in the shop window, and at any place, it should be able to show its front side.

3. The product has the size of a bag or a pair of shoes.

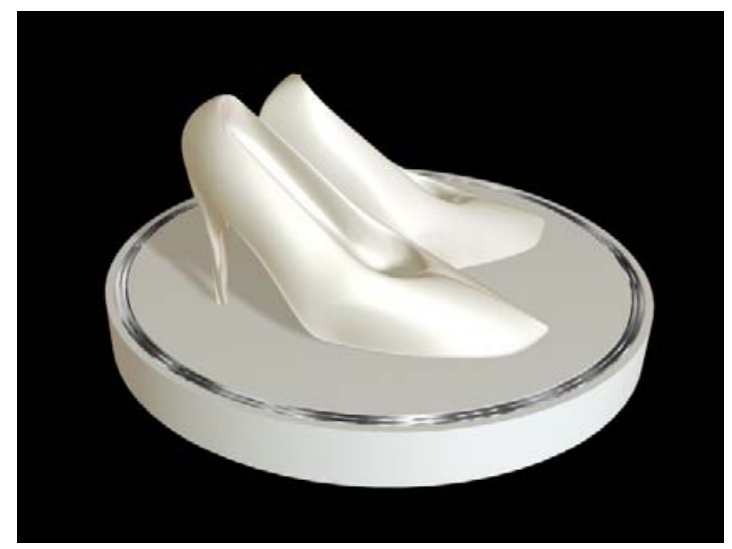

Fig. 2. 3D model of the turntable with a pair of shoes 
A dedicated turntable was designed and manufactured. After consideration of various options, a cardan suspension was chosen. This suspension is a universal mounting which, by means of gimbals, allows the supported part to remain horizontal irrespective of the orientation of the rest of the instrument. This sort of mounting is often seen in compasses in old ships. In this case, the mounting is turned up side down, so the ground is the horizontal fixture and it is possible to position the upper part of the mounting in any desired angle. This results in a $35 \mathrm{~cm}$ wide turntable that is $7 \mathrm{~cm}$ high. This size was chosen because the width ensures that it fits a pair of shoes and the height makes sure the shoes can be tilted for 20 degrees. The base part and the disc on top of the turntable are made of a plastic. The two inner rings are made of aluminum because more strength was needed to withstand the forces that would be put on them. The total weight of one turntable is about 8 kilos.

The positioning of the 2 rings and the disc is done with 3 servos: one for every angular degree of freedom. The model of the turntable is given in figure 2 . To fit the turntable in the high-fashion look of the shop window, the plastic that is used is spraypainted with glossy white paint. The aluminum parts keep their original metal color. In total 3 identical turntables were manufactured.

The actuation of the servo motors is controlled with Phidgets ${ }^{\mathrm{TM}}$ [6]. In every turntable one control unit is built which is connected to the controlling pc. The control pc is on its turn connected to the gaze tracker and to the touch interface, so when a user selects a product on a turntable (either with gaze or touch), the 3 servos are actuated and turn the product towards the user. At the time of testing, the servos move from their starting position to their end position at a constant speed.

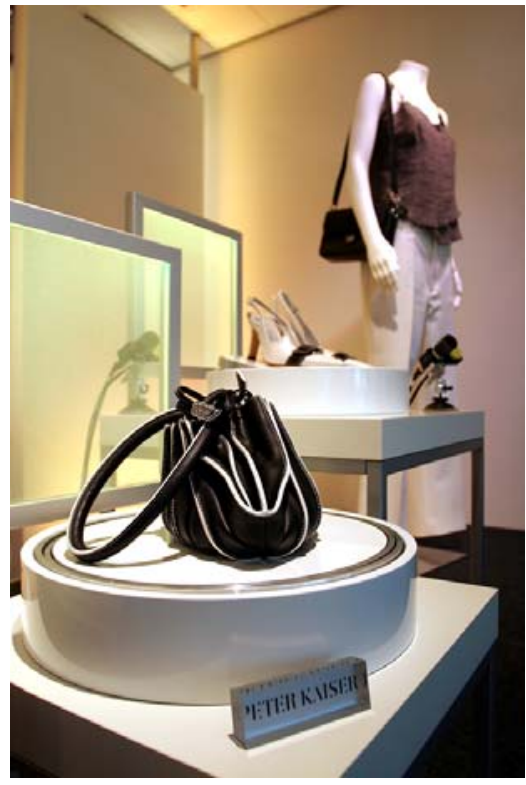

Fig. 3. Combined feedback mechanisms

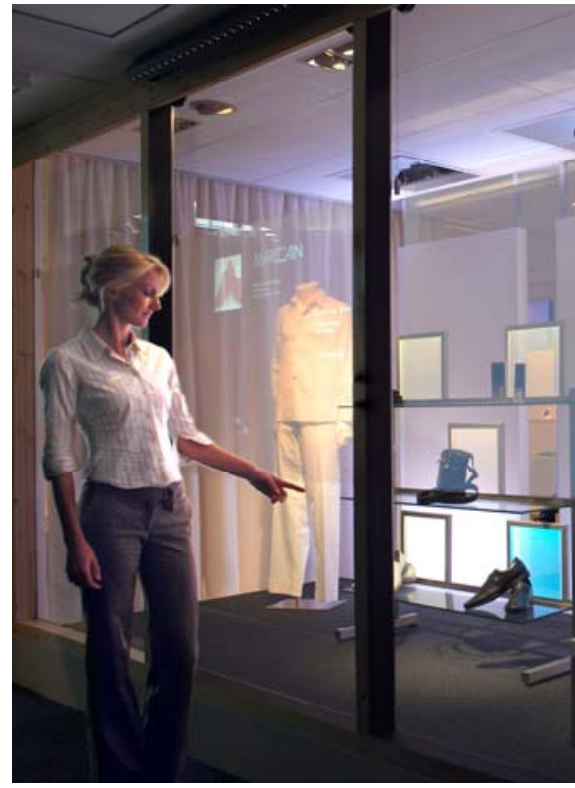

Fig. 4. Touch interaction 


\section{Feedback method: Light 'em up}

In many everyday interaction mechanisms, light is a powerful feedback from the system to the user. Lights also attract attention. This also holds for shop windows: the best lit products get the most attention. [2,3].

Hence, light feedback is also currently implemented in the Intelligent Shop Window. When a shopper looks at a product or even points at it, a light behind the product changes color and keeps the attention of the user focused on the product. It gives the shopper feedback that the system knows where the interests of the shopper are at that particular moment. When the user walks away, or indicates that he/she is interested in something else in the shop window, the light returns to its regular state.

To be able to test both feedback mechanisms without altering the experimental setup, the two mechanisms are combined as indicated in figure 3 . Three tables of different sizes and height are placed in the shop window. The turntables are placed in the middle on top of them and the light tiles are mounted at the back of the tables in such a way that the bottom of the light tile is at the same height as the top of the turntable.

\subsection{Personal Interaction}

In the personal interaction phase described in Vogel et al. [1], gesture and touch interaction is used. In the current ISW, both gaze and touch interaction can be used in this phase. A user can select products with gaze, but also point at them on the glass and browse through the information displayed on the screen using touch interaction. Here, gaze and touch interaction are the most logical interaction styles to use, because the user is standing close to the window which is already used as a screen. In contrast, other interaction styles like speech are less natural or useful in a noisy shopping environment.

When a user walks away after using the interface on the screen, the information that is displayed disappears and another interaction phase can be entered.

\section{Evaluation of Interaction and Feedback Mechanisms}

To be able to evaluate the two interaction methods and two feedback mechanisms described in the previous chapter, an evaluation with users has been done. In the following sections, the participants, setting, design, procedure and results will be discussed.

\subsection{Participants, Setting and Design}

The experiment involved 18 participants, ( 9 women and 9 men). Participants were selected in the range from 23-40 years old. The mean age was 31 . They all indicated they go shopping from time to time. Participants were randomly assigned to the predefined sequence of the experimental conditions.

The experiment took place in front of the ISW of the ShopLab at the Philips High Tech Campus. Participants would be asked to step in front of window 3 (out of 4) of the shop window, whereas the experimenter would stand in front window 2, starting and ending the tasks with the touch interface on the window.

A camera was used to record the whole experiment and a small unobtrusive microphone was placed between the experimenter and the participant. The products in the 
shop window were dedicated female products for female participants and dedicated male products for male participants.

We adopted a within-subjects design in which the method of interaction and the feedback mechanism in the shop window was manipulated, as indicated in table 1 . There were in total six conditions. We were interested in comparing gaze and touch interaction, as well as comparing the different feedback mechanisms.

Table 1. Interaction and feedback conditions

\begin{tabular}{|c|c|c|c|}
\hline & $\begin{array}{c}\text { Turntable } \\
\text { feedback }\end{array}$ & $\begin{array}{c}\text { Light tile } \\
\text { feedback }\end{array}$ & No feedback \\
\hline $\begin{array}{c}\text { Gaze } \\
\text { interaction }\end{array}$ & 1. & 2. & 3. \\
\hline $\begin{array}{c}\text { Touch } \\
\text { interaction }\end{array}$ & 4. & 5. & 6. \\
\hline
\end{tabular}

The experiment consisted of 3 parts, in which participants were requested to perform several tasks.

- In the first part, participants would either use gaze or touch interaction (the order was balanced over user) and perform 3 tasks. A typical task in the experiment was: "Find the price of the white shoes in the shop window". By either looking at the product or pointing at it and using the touch interface, participants were able to find the requested information about the product. At the moment the product was 'selected', typical information about that product appeared on the glass of the shop window. We opted for three tasks in order to try out the two feedback conditions and the no feedback condition. Each tasks was then accompanied by a different form of feedback: 1. no feedback, 2. lighting the tile, 3. turning the product. After completing the tasks, the participant was asked to fill in a questionnaire about the interaction style that was used. This questionnaire enclosed multiple questions on hedonic values, perceived ease of use, perceived usefulness and affect (see §3.2).

- In the second set, again three tasks were carried out. The interaction style that was used changed but the feedback methods were not changed. After the three tasks the participant filled in the same questionnaire, but now about the interaction style in the second set of tasks.

- In the third set of tasks, participants could choose their preferred interaction style (touch or gaze interaction) and they were asked to elaborate on their choice. Only two tasks were given and only the feedback mechanisms 'light tile' and 'turntable' were presented. Before starting the third set of tasks, the experimenter told the participants that in the shop window two different feedbacks would be given and that questions would be asked about them after the tasks. After completing the two tasks, participants would be asked to fill in a questionnaire, directly comparing the light tile and turntable feedback mechanisms.

- A closing interview was done with all participants. 


\subsection{Measures}

A multiple set of measures was used to test both the direct effects of the feedback mechanisms inside the shop window as well as the speed, effectiveness and ease of use of the interaction styles.

Time measurements. The time between the start and end of a task was measured.

The Unified Theory of Acceptance and the Use of Technology (UTAUT) [7,8,9] is a measure of technology acceptance. The UTAUT was used with some adaptations for the shopping domain. The scales perceived ease of use (PEOU) and perceived usefulness (PU) are adopted in this experiment.

The Affect scale (AF) [10] refers to the affective emotional considerations about the interaction.

The Hedonic part of the HED/UT scale of van der Heijden [11] which includes factors as enjoyability and fun.

The Users' preference for a feedback mechanism is a list of carefully composed questions, including comparing questions between the two feedback mechanisms, whether or not the response of the system is noticed and if it is and adequate feedback for interaction with the intelligent shop window.

An interview was done with all the participants about their general preference of interaction method and feedback mechanism. Also some questions about privacy (cameras) were asked.

\subsection{Procedure}

In order to calibrate the gaze tracker to each individual participant, a profile of the participants face had to be made. Because this profiling of participants was time consuming (approx. 1 hour), this occurred at least 3 days before the actual experiment. of time consuming profiling (approx. 1 hour), participants were asked to take part in the real experiment another time.

At the start of the experiment itself, the participants were again welcomed and they filled in an informed consent form, approving audio and video recordings. They were also explained what the purpose of Shoplab was and the goal of the experiment. Next, the participant was asked to step in front of window $\mathrm{nr} 3$. At the glass of the window a large button was projected labelled 'START'. The experimenter stepped in front of the window next to the participant (window 2). On this window, the participant number could be chosen. With this choice, the sequence of interaction styles and feedback mechanism was also chosen which would be presented to the participant. The order of these sequences was balanced out over all participants.

After explaining that the interaction style with the interactive shop window was under investigation and not the actions of the participant, it was explained which interaction style should be used during the first tasks (touch or gaze interaction). Now, the first task of the first set was read out loud to the participant. After the task was read, the participant pressed the 'start' button on the touch-sensitive shop window (starting the time measurement) and carried out the task. When the participant read 
the correct answer out loud, the experimenter stopped the time measurement by pressing a button on window 2. After carrying out the three tasks of the set, the participant was asked to fill in a questionnaire about the interaction style that was just used. The second set of tasks was carried out in the same manner, with filling in the questionnaire shortly after that.

Before starting the third set of tasks, the preferred interaction style of the participant was indicated on window 2 by the experimenter. The participant was asked to pay close attention to what was happening inside the shop window when interacting with it. Then, the two tasks were carried out and again a questionnaire was filled in about the feedback mechanisms in the shop window. Afterwards, this questionnaire was used as a basis for an interview where the participants gave their impression of the experiment and the thoughts they had about the interaction styles and the feedback mechanisms.

\subsection{Results}

\section{Hedonic scale}

All questionnaires included the Hedonic part of the HED/UT scale by van der Heijden [9]. A T-test was used and Cronbach's alpha was calculated for the whole scale: $\mathrm{a}=0,92$, indicating the set of items measures a single unidimensional latent construct. This calculation included all ratings from all participants on touch and gaze interaction and on the 2 feedback mechanisms.

Only the sub-item 'Boring/Fun' in the Hedonic scale shows a significant difference between touch and gaze interaction, meaning that touch was rated more fun than gaze. Also the item 'Disgusting/Delightful' shows a marginal significant result. On all the other items, no significant difference was found. Despite the significant difference in fun between the interaction styles, for the complete HED scale no significant difference was found. However, given the fact that touch interaction is known to many participants and that none of them ever worked with gaze interaction, this non-significant difference between the interaction styles is still a striking result.

In the results for the feedback mechanisms, in all cases, the median score is for the turntable feedback one point higher than the light tile rating. Also the variance for the turntable feedback is smaller than the variance in rating for the light tile, so participants were quite agreed in their opinions regarding the turntable.

Half of the items (dull/exciting, boring/fun, serious/playful, unthrilling/thrilling) in the HED scale indicate a significant difference $(\mathrm{p}<0.05)$ between the turntable and the light tile, in favor of the turntable. All other items except the 'disgusting/delightful' item show a strong trend that the turntable is rated higher than the light tile. The results for the complete HED scale for the feedback mechanisms show a marginally significant difference in favor of the turntable $(p=0,052, \mathrm{z}=-1,941, \mathrm{n}=18)$, so on the Hedonic scale, participants rated the turntable feedback higher than the light tile.

\section{Perceived Ease of Use and Perceived Usefulness ${ }^{2}$}

Next, the data for the scales 'perceived Ease of Use' (PEOU), 'perceived usefulness' (PU) and 'affect' (AF) is analyzed. The calculated Cronbach's alpha for PEOU is: $\alpha=0,943$ and for PU: $\alpha=0,791$.

In figure 5 it is striking to see that the mean of all answers of all participants on touch interaction is higher on all items in the PEOU and PU scale. For PU a higher variance 
Gaze vs. Touch Interaction

Perceived Ease of Use (PEOU) and Perceived Usefulness (PU)

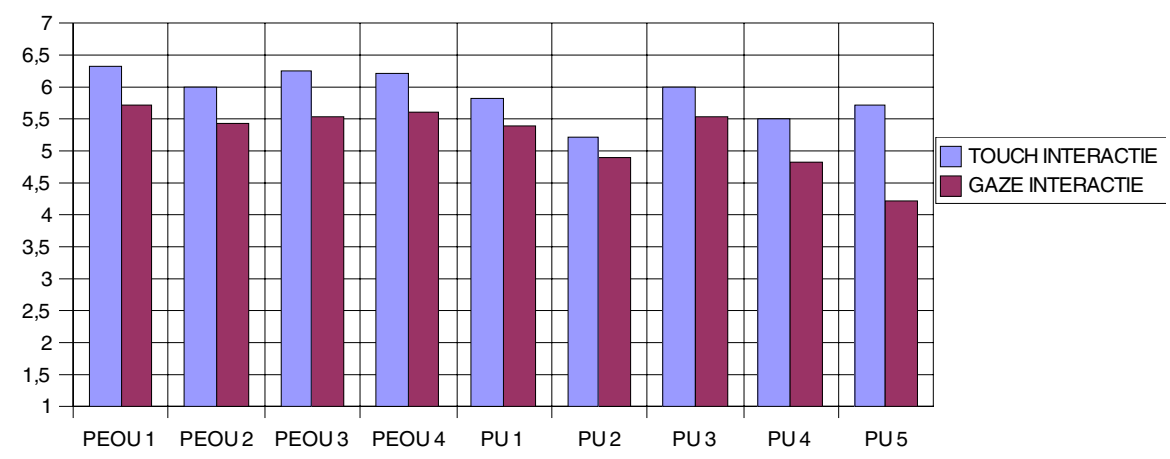

Fig. 5. Mean results for PEOU and PU scales for touch and gaze interaction

for gaze interaction is observed, but also that the median ratings for gaze are lower than for touch. A Wilcoxon signed ranks test is performed to see if this difference is significant. The difference in PU between touch and gaze interaction was only significant for PU5 ( $\mathrm{z}=-2.687, \mathrm{df}=17, p<0.05)$ indicating that participants rated the amount of control he/she had in operating the system higher with touch interaction. In total, touch interaction scores are significantly higher on perceived usefulness than gaze interaction.

The difference in PEOU between touch and gaze interaction was not significant. Not a single item scores significantly different in favor of touch or gaze, although the trend points out that touch interaction scores higher on Ease of Use.

\section{Affect}

From figure 6 it is clear that touch interaction scores higher on all items in the Affect scale. A Cronbach's alpha for the scale was calculated: $\alpha=0,889$. The variance for affect on gaze interaction is larger than for touch interaction.

\section{Gaze vs. Touch interaction Affect}

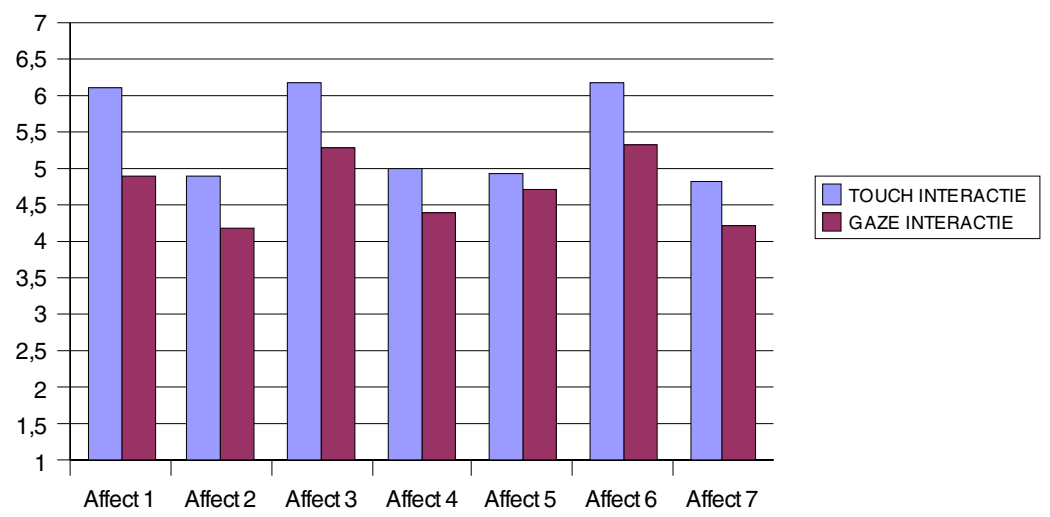

Fig. 6. Mean results for Affect scale for touch and gaze interaction 
The difference in affect between touch and gaze interaction was in four out of seven items significant $(p<0.05)$ indicating that participants enjoyed working with touch interaction more than working with gaze. Some items in the affect scale are 'frustration' and 'awkwardness' of the interaction style. Also, regarding the complete affect scale the following result was obtained: $p=0.007, \mathrm{z}=-2.676, \mathrm{n}=17$, meaning that touch interaction scores significantly higher on affect than touch interaction.

\section{Users' preference for a feedback mechanism}

All users were asked to compare the two feedback mechanisms in the shop window with each other. In figure 7, the ratings are given for the 5 questions that are stated below. It is clear that the turntable is rated higher than the light tile on all questions. Also, when the light tile and the turntable were directly compared with the question 'Which form of feedback do you prefer?', 12 out of 18 participants answered they preferred the turntable.

1. I noticed that [sometimes lights went on/the products in the shop window were sometimes moving].

2. [A light behind the product/Moving a product] in the shop window is an adequate response of the system.

3. I find [a light behind the product/movement of the product] in the shop window useful

4. [A light behind a product/Movement of the product] is helpful for me in searching the shop window

5. [A light behind a product/Movement of the product] enhances my effectiveness in searching.

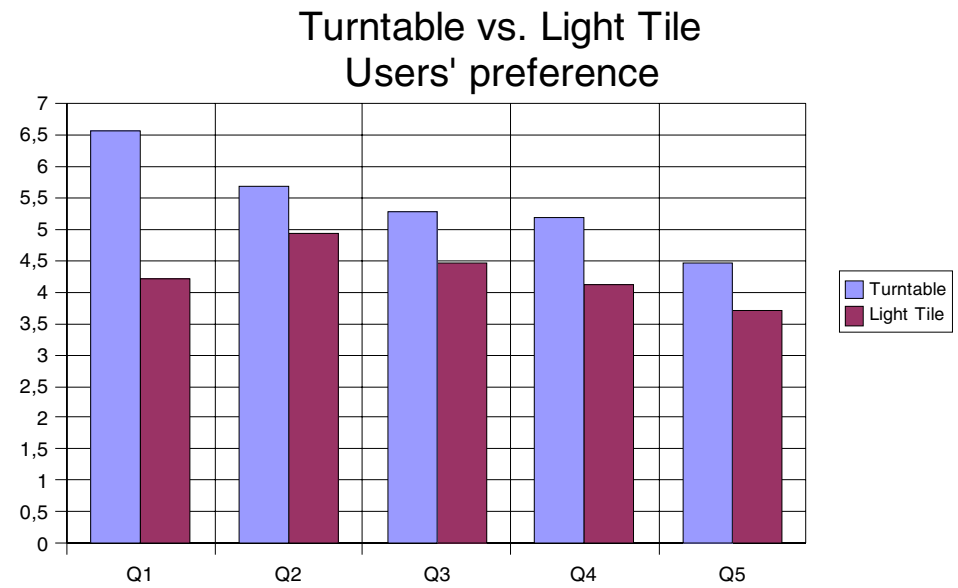

Fig. 7. Turntable vs. Light Tile, users' preference results

The opinions on the light tile were wider spread than opinions on the turntable. One explanation for this is that 5 out of 18 participants indicated that they did not see the light tile while performing the tasks. Only when they were explicitly instructed to look at the light tile, they saw the effect. A Cronbach alpha is calculated: $\alpha=0.573$, so 
all items in this set were treated individually. Only the question if the feedback was noticed by the user during the tasks was significant in favor of the turntable $(\mathrm{Z}=$ $3,078, \mathrm{df}=18, p<0,05)$. The other questions were not significant, although the trend showed that users had a slightly higher preference for the turntable.

\section{Time measurements}

During all the tasks in the experiment, a time measurement was done to get an indication on which interaction style gave the fastest results. The timer was started when the task was read out loud by the experimenter and the participant pushed the 'start' button on the glass of the shop window. When the correct solution to the task was read out loud by the participant, the timer was stopped by the experimenter. The participants were not aware that a time measurement was done. Per participant, 8 time measurements were done. 3 for every interaction style and another 2 with the interaction style of the participants' choice. For this comparison only the first three tasks for both interaction styles were investigated, because of a large learning effect.

For 7 out of 18 participants, the task that was performed fastest was with gaze interaction, for the remaining 11 participants this fastest task was performed with touch interaction. A mean time for carrying out a task was around 10 seconds. The tasks where it took longer than 30 seconds to complete were excluded from the data, because the participant was lost in the interface on the glass (using touch) or the gaze tracker did not work properly. When a task took longer than a minute, the task was stopped by the experimenter and skipped. A total of 6 records for gaze and 3 records for touch interaction were excluded because of the stated reasons. This exclusion was an indication that gaze interaction did not work as stable as touch interaction. With the data exclusion taken into account, for 10 participants, the mean time for the tasks with gaze interaction is shorter than the mean time for tasks with touch interaction. This result showed that gaze and touch interaction were very competitive in interaction speed, but it has to be emphasized that the participants were not trying to complete the tasks as quick as possible, nor were they told that they were going to be timed. To be conclusive on which interaction style works the fastest, another test has to be done, focusing on the time it takes to complete a task.

\section{Conclusion}

From the user study it became clear that participants think that gaze interaction is quite fun to work with, but from the interview it became clear that the loss of control is for many difficult to accept. Also it seemed to be difficult to find a solution when an unexpected or no response was given by the gaze system. Touch interaction is found to be robust and participants knew how to operate it immediately. However, drawbacks related to the public shopping environment (vandalism, fingerprints on the window) were mentioned very often.

Regarding our first research question: What is the best way to interact with a large shop window? we can conclude that touch interaction is at this moment a very robust and intuitive interaction method. However, when gaze tracking technology has become robust and stable and does not require a profile of a face anymore to be able to track it, gaze interaction can be a very attractive, vandalism proof and powerful interaction style in shop windows. 
Regarding the second research question: What is the best form of feedback that gives the user a clear and understandable interaction experience? we can conclude that both feedback mechanisms that were investigated, provided the user with clear and understandable feedback that the system responded to the user. However, the polite products concept was rated higher than the light tile and especially in combination with gaze interaction, this form of feedback is a very strong one in the context of shopping environments. Shoppers do not need to touch anything to take a good look at a real product in a show window. The product automatically presents itself politely when looked at.

\section{References}

1. Vogel, D., Balakrishnan, R.: Interactive public ambient displays: transitioning from implicit to explicit, public to personal, interaction with multiple users (2004)

2. van Loenen, E., Lashina, T., van Doorn, M.: Interactive Shop Windows. In: Ambient Lifestyle: From Concept to Experience. Bis Publishers, Amsterdam (2006)

3. van Loenen, E., Lashina, T., van Doorn, M., van Gelder, K., Teeven, V., de Bruin, W., van Haasen, R.: DreamScreen: Intelligent Shop Window. In: New Business through User Centric Innovation, Philips Research Europe publication, ISBN 907444573X

4. Maglio, P.P., Barrett, R., Campbell, C.S., Selker, T.: SUITOR: an attentive information system (2000)

5. Vertegaal, R.: Principles \& methodology: Designing attentive interfaces (2002)

6. Phidgets, http://phidgets.com/

7. Davis, F.D.: A technology acceptance model for empirically testing new end-user information systems: Theory and results (1986)

8. Davis, F.D., Bagozzi, R.P., Warshaw, P.R.: User acceptance of computer technology: A comparison of two theoretical models. Management Science (1989)

9. Venkatesh, V., Morris, M.G., Davis, G.B., Davis, F.D.: User acceptance of information technology: Toward a unified view (2003)

10. Keinonen, T.: Expected usability and product preference (1997)

11. Van der Heijden, H.: User Acceptance of Hedonic Information Systems (2004) 